\title{
Universality of the self gravitational potential energy of any fundamental particle
}

\author{
Antonio Alfonso-Faus \\ Escuela de Ingeniería Aeroespacial \\ E.U.I.T. Aeronáutica, Universidad Politécnica de Madrid \\ Plaza Cardenal Cisneros 3, 28040 Madrid, Spain \\ e-mail: aalfonsofaus@yahoo.es
}

\begin{abstract}
Using the relation proposed by Weinberg in 1972, combining quantum and cosmological parameters, we prove that the self gravitational potential energy of any fundamental particle is a quantum, with physical properties independent of the mass of the particle. It is a universal quantum of gravitational energy, and its physical properties depend only on the cosmological scale factor $\mathrm{R}$ and the physical constants $\hbar$ and c. We propose a modification of the Weinberg's relation, keeping the same numerical value, but substituting the cosmological parameter $\mathrm{H} / \mathrm{c}$ by $1 / \mathrm{R}$.
\end{abstract}

Keywords: Cosmology, quantum mechanics, gravitational energy, cosmological scale factor, Weinberg's relation.

In 1972 Weinberg [1] advanced a clue to suggest that large numbers are determined by both, microphysics and the influence of the whole universe. He constructed a mass using the physical constants G, $\hbar, \mathrm{c}$ and the Hubble parameter $\mathrm{H}$. This mass was not too different from the mass of a typical elementary particle and is given by

$$
\mathrm{m} \approx\left(\hbar^{2} \mathrm{H} / \mathrm{Gc}\right)^{1 / 3}
$$

We consider also a general elementary particle of mass $\mathrm{m}$. The self gravitational potential energy $\mathrm{E}_{\mathrm{g}}$ of this quantum of mass $\mathrm{m}$ (and size its Compton wavelength $\hbar / \mathrm{mc}$ ) is given by 


$$
\mathrm{E}_{\mathrm{g}}=\mathrm{Gm}^{2} /(\hbar / \mathrm{mc})=\mathrm{Gm}^{3} \mathrm{c} / \hbar
$$

Combining (1) and (2) we can eliminate the mass $m$ to obtain

$$
\mathrm{E}_{\mathrm{g}} \approx \hbar \mathrm{H}
$$

This expression has an important quantum-cosmological interpretation. We know today that the cosmological scale factor $\mathrm{R}$ is of the order of ct, $\mathrm{t}$ the age of the universe [2]. In this reference [2] the cosmological scale factor $\mathrm{R}$ is obtained in terms of the cosmological time $t$ as

$$
\mathrm{R}(\mathrm{x}) / \mathrm{R}(1)=[2 \mathrm{x} /(3-\mathrm{x})]^{2 / 3}
$$

where $x=t / t_{0}$ is the dimensionless parameter for cosmological time in terms of the present age of the universe $t_{0}$. For $t=t_{0}$ we have $x=1$. $R(1)$ in (4) is the present value of the cosmological scale factor. The following fig. 1 gives the graphical plot of this cosmological scale factor $\mathrm{R}(\mathrm{x})$ :

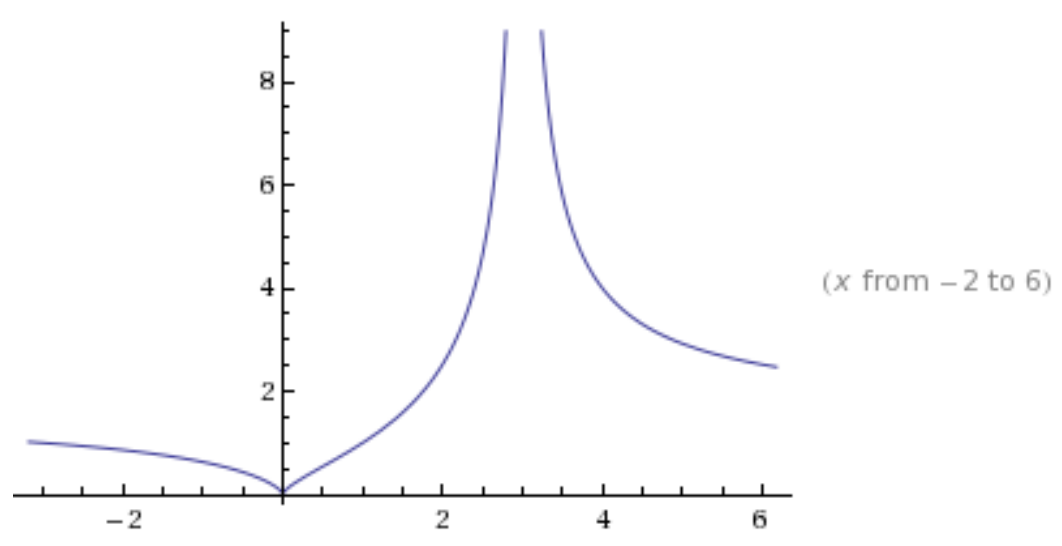

Fig.1 Cosmological scale factor $\mathrm{R}(\mathrm{x}) / \mathrm{R}(1)$ versus time $\mathrm{x}=\mathrm{t} / \mathrm{t}_{0}$ 
In this plot of $\mathrm{R}(\mathrm{x})$ versus $\mathrm{x}$ we see that there is an almost linear expansion law from $x=0$ to about $x=1.4$. A series Taylor expansion of (4) around $x$ $=1$ (today) gives

$$
\mathrm{R}(\mathrm{x}) / \mathrm{R}(1)=1+\mathrm{R}^{\prime}(1) / \mathrm{R}(1)(\mathrm{x}-1) / 1_{i}+\mathrm{R}^{\prime \prime}(1) / \mathrm{R}(1)(\mathrm{x}-1)^{2} / 2 i+\ldots
$$

Substituting the derivatives of $\mathrm{R}(\mathrm{x})$ from (4) into (5) we get

$$
\mathrm{R}(\mathrm{x}) / \mathrm{R}(1) \approx \mathrm{x}+(\mathrm{x}-1)^{2} / 4+\mathrm{O}(\mathrm{x}-1)^{3}
$$

The speed of expansion is then

$$
\mathrm{R}^{\prime}(\mathrm{x}) / \mathrm{R}(1) \approx 1+(\mathrm{x}-1) / 2+\mathrm{O}(\mathrm{x}-1)^{2}=(1+\mathrm{x}) / 2+\mathrm{O}(\mathrm{x}-1)^{2}
$$

The acceleration is then

$$
\mathrm{R}^{\prime \prime}(\mathrm{x}) / \mathrm{R}(1) \approx 1 / 2+\mathrm{O}(\mathrm{x}-1)
$$

From the definition of the Hubble parameter $\mathrm{H}$ we have

$$
\mathrm{H}(\mathrm{x})=\mathrm{R}^{\prime}(\mathrm{x}) / \mathrm{R}(\mathrm{x}) \approx[(1+\mathrm{x}) / 2] / \mathrm{x}
$$

Taking into account $\mathrm{dx}=\mathrm{dt} / \mathrm{t}_{0}$ we have $\mathrm{H}(\mathrm{t})$ with its dimension $1 /$ time

$$
\mathrm{H}(\mathrm{t}) \approx 2 /\left(\mathrm{t}+\mathrm{t}_{0}\right)
$$

and from here we get for the present time $H\left(t_{0}\right) \approx 1 / t_{0}$. Present values for the age of the universe give $\mathrm{t}_{0} \approx 1.3710^{10}$ years and therefore $\mathrm{H}\left(\mathrm{t}_{0}\right) \approx 71.4$ $\mathrm{Km} /(\mathrm{sec} \mathrm{Mpc})$. This is well in the range of observations [3]. The Hubble radius for today is defined as $\mathrm{c} / \mathrm{H}\left(\mathrm{t}_{0}\right) \approx \mathrm{ct}_{0}$ and taking this radius as the 
present reference for the cosmological scale factor, i.e., $\mathrm{c} \mathrm{t}_{0} \approx \mathrm{R}(1)$ we get from (6), (7) and (8) to first order

$$
\mathrm{R}(\mathrm{t}) \approx \mathrm{ct} ; \mathrm{R}^{\prime}(\mathrm{t}) \approx \mathrm{c} ; \mathrm{R}^{\prime \prime}(\mathrm{t}) \approx 0+\mathrm{c} /\left(2 \mathrm{t}_{0}\right)
$$

The expression for $\mathrm{R}^{\prime \prime}(\mathrm{t})$ proves that there is a second order effect in the expansion of the universe, the acceleration $\mathrm{c} / 2 \mathrm{t}$. It has gone unnoticed up to a few years ago, [4] and [5]. The reason is here evident: it is a second order effect.

The central point of the result in (11) is that the ratio $\mathrm{H} / \mathrm{c}$ is of the order of the inverse of $\mathrm{R}(\mathrm{t})$, at least close to the present time. In other words, following the same large number approach of Weinberg [1], we can use $1 / \mathrm{R}$ instead of $\mathrm{H} / \mathrm{c}$ in its formulation. Substituting in (1) we get our revised Weinberg relation as

$$
\mathrm{m} \approx\left(\hbar^{2} / \mathrm{GR}\right)^{1 / 3}
$$

Hence, substituting $\mathrm{H} / \mathrm{c} \approx 1 / \mathrm{R}$ in (3) we get the important result

$$
\mathrm{E}_{\mathrm{g}} \approx \hbar \mathrm{c} / \mathrm{R}
$$

This is the Planck-Einstein equation. In 1923, Louis de Broglie postulated that the Planck constant $\hbar$ represents the product of the momentum and the quantum wavelength of not just the photon, but any particle. This was confirmed by experiments soon afterwards. Then we can interpret $R$ in (13) as the wavelength of the quantum of gravitational energy. It is unlocalized, as the energy of the gravitational field [6]. And its momentum $p_{g}$ is then 


$$
\mathrm{p}_{\mathrm{g}}=\mathrm{E}_{\mathrm{g}} / \mathrm{c}=\hbar / \mathrm{R}
$$

The equivalent expressions (3) and (13) for $E_{g}$ have very important implications. First, the elimination of the mass $\mathrm{m}$ of the elementary particle, any fundamental particle in fact, clearly makes the gravitational potential energy of any particle a universal expression. The presence of $\hbar$ in the expressions (3) and (13), and the cosmological parameters $H$ or $\mathrm{R}$, implies that, with the same aim as Weinberg had [1], we have obtained a universal relation between the physical constants, $G, \hbar$, and a cosmological parameter R (12). Second, the expression (13) can be interpreted as being a quantum of energy $E_{g}$, and it certainly has a gravitational nature as given by the initial concept used in (2): a gravitational potential energy. With these results we advance the following conjecture:

The quantum of gravitational energy is given by the expression $E_{g}=\hbar c / R$, it has a wavelength of the order of the size of the universe, $R$, and a momentum $p_{g}=\hbar / R$. Its equivalent mass has a value $m_{g}=\hbar / c R \approx 10^{-66}$ grams, a figure found in many different instances in the scientific literature.

The present knowledge of the composition of the universe is roughly $4 \%$ of normal baryon matter (stars and galaxies), $24 \%$ of non-baryon dark matter (inferred from its gravitational action on stars in galaxies, clusters of galaxies etc.) and 72\% dark energy, from indirect arguments. All this mass (energy) must imply gravitation in some way, and therefore it represents an 
amount of gravitational energy corresponding to a total estimated mass for the universe of about $\mathrm{M} \approx 10^{56}$ grams. Then, regardless of its origin, the total amount of gravity quanta should be $\mathrm{MR} / \hbar \mathrm{c} \approx 10^{122}$.

The gravitational potential energy of the universe is of the order of $\mathrm{Mc}^{2} \approx$ $10^{77} \mathrm{ergs}$. On the other hand the energy of the quantum of gravitation found here is $\mathrm{E}_{\mathrm{g}}=\hbar \mathrm{c} / \mathrm{R} \approx 10^{-45}$ ergs. Dividing we get a number $\mathrm{n} \approx 10^{122}$. This is a well known number. It may be linked to the entropy of the universe (with Boltzmann constant $\mathrm{k}=1$ ). It is also the order of magnitude of the discrepancy between the values of the cosmological constant $\Lambda$, as derived from cosmological information, and from the standard particle theory [7]. Here it may be thought as been a quantum number representative of the state of the universe as it is today. And there are $\mathrm{n} \approx 10^{122}$ possibly entangled gravity quanta. This gives a pressure, and a corresponding energy density, that is well within the current known values for the seeable universe (with Hubble radius).

\section{Acknowledgement}

I am thanking the owners of the Wolfram Mathematica Online Integrator that I have used to obtain the Figure 1 in this work. 


\section{REFERENCES}

[1]. Weinberg, S., Gravitation and Cosmology: Principles and Applications of the General Theory of Relativity, page 619, John Wiley \& Sons, Inc., (1972).

[2]. Alfonso-Faus, A., (2011) "Evidence for a disaggregation of the universe", arXiv: 1104.3781.

[3]. Hinshaw, G. et al. (WMAP Collaboration). (feb 2009). "FiveYear Wilkinson Microwave Anisotropy Probe Observations: Data Processing, Sky Maps, and Basic Results" (Table 7). The Astrophysical Journal Supplement 180 (2): 225-245. arXiv: 0803.0732 .

[4]. Riess, A. G., et al. [Supernova Search Team Collaboration] Astron. J. 116, 1009 (1998) [arXiv: astr-ph/9805201].

[5]. Perlmutter, S., et al. [Supernova Cosmology Project Collaboration], Astrophys. J. 517, 565 (19999 [arXiv: astro-ph/9812133].

[6]. Misner, C.W., Thorne, K.S., and Wheeler, J.A., GRAVITATION, page 466: "WHY THE ENERGY OF THE GRAVITATIONAL FIELD CANNOT BE LOCALIZED", Freeman and Company, San Francisco, (1973).

[7]. Alfonso-Faus, A., "Artificial contradiction between cosmology and particle physics: the lambda problem", arXiv: 0811.3933 and Astrophys. Space Sci. 321: 69-72, (2009) 